\title{
Fine motor assessment with unsupervised learning for personalized rehabilitation in Parkinson Disease*
}

\author{
E. Rovini, L. Fiorini, D. Esposito, C. Maremmani and F. Cavallo, Member, IEEE
}

\begin{abstract}
Parkinson disease (PD) is a common neurodegenerative disorders characterized by motor and non-motor impairments. Since the quality of life of PD patients becomes poor while pathology develops, it is imperative to improve the identification of personalized rehabilitation and treatments approaches based on the level of the neurodegeneration process. Objective and precise assessment of the severity of the pathology is crucial to identify the most appropriate treatments. In this context, this paper proposes a wearable system able to measure the motor performance of PD subjects. Two inertial devices were used to capture the motion of the lower and upper limbs respectively, while performing six motor tasks. Forty-one kinematic features were extracted from the inertial signals to describe the performance of each subjects. Three unsupervised learning algorithms (kMeans, Self-organizing maps (SOM) and hierarchical clustering) were applied with a blind approach to group the motor performance. The results show that SOM was the best classifier since it reached accuracy equal to 0.950 to group the instances in two classes (mild vs advanced), and 0.817 considering three classes (mild vs moderate vs severe). Therefore, this system enabled objective assessment of the PD severity through motion analysis, allowing the evaluation of residual motor capabilities and fostering personalized paths for PD rehabilitation and assistance.
\end{abstract}

\section{INTRODUCTION}

Parkinson Disease (PD) is a complex neurodegenerative disorder caused by a critical deficiency of dopamine in the brain, which afflicts approximately 6.5 millions of people worldwide, with increasing incidence rate [1], and high related costs [2]. The pathology is strongly disabling for affected patients due to both motor and non-motor symptoms [3]. Even if the knowledge of PD has increased significantly over the recent years, the current methods for diagnosis, disease progression monitoring and rehabilitation remain suboptimal for PD management [4].

According to the "Chronic Care Model" [5], to properly manage chronic diseases it is important to have a predefined

${ }^{*}$ Research supported by SI-ROBOTICS Ministero dell'Istruzione, dell'Università e della Ricerca (Funding Programme PNR 2015-2020).

E. Rovini, L. Fiorini, and F. Cavallo are with Scuola Superiore Sant'Anna, Pisa, Italy (corresponding author Filippo Cavallo; phone: +390597 672152; e-mail: filippo.cavallo@santannapisa.it). path that involves a continuous multidisciplinary assistance, to allow long term personalized care-rehabilitation plan [6]. It is important therefore to organize all actions according to the level of disease and related needs, guaranteeing right management path for every patient. These actions should be personalized according to the severity of the disease and the complexity of the patient, considering also a standardization in terms of costs and quality of care [7].

Due to the nature of PD (chronic with a progressive worsening), patients should periodically undergo through neurological examinations for assessment of level of neurodegeneration. Therefore, the possibility to objectively assess their motor performance is a first step to evaluate their residual capabilities and, consequently, to address them towards opportune rehabilitative and assistive paths.

Recently, research groups have worked to objectively measure the motor performance of the patients, since the motor symptoms are those that generally lead the neurologist to the diagnosis [3]. A study revealed that the overall classification rate is not only limited by technical accuracy but also by clinical accuracy [8]. Indeed, a method to objectify the motion can lead to a quantitative diagnosis of the PD, overtaking the problems linked to the subjectivity and to the inter-rater and intra-rater variability, thus increasing the accuracy of the diagnosis.

Over the last decade, Inertial Measurement Units (IMUs) were used to measure motor performance [9]. However, the literature has a lack of consensus regarding the optimal number of sensors, and the optimal site for their placement, for the assessment of PD motor symptoms [10]. Moreover, to provide automatic assessment of the performances measured, thus discriminating PD patients from healthy subjects or other pathologies, recent works implemented different machine learning approaches [11], such as Support Vector Machine [12], Naïve Bayes, k-NN, Random Forest [13], Decision Tree, and Linear Discriminant Analysis [14]. However, supervised and semi-supervised algorithms needs to be trained with a large amount of data prior to be used as a decision support tool for diagnostic and rehabilitative purposes. Additionally, these methods need data annotation that require a large amount of time and it is not suitable for daily use.

Unsupervised learning has been recently used in several works for activity recognition purposes [15], [16]. Clustering

D. Esposito is with Co-Robotics srl, Capannoli, Pisa, Italy (e-mail: dario.esposito@corobotics.eu).

C. Maremmani is with AUSL Toscana Nord Ovest (e-mail: carlo.maremmani@uslnordovest.toscana.it). 
techniques are defined as "the art to find groups in data", since it has the potential to identify similarity and differences among instances in the features spaces independently from their label [17]. Indeed over the last years, these techniques were used to identify physical behavior changes in common daily motor tasks [18] and to investigate similarity and differences in daily behavioral pattern of healthy older adults and person with cognitive impairments [19]. Recently, cluster techniques were also used to identify healthy subjects with behavioral health risk profiles [20]. Nevertheless, to the best of authors' knowledge, using data from motion analysis for clustering of PD patients according to the gravity of the diseased represents a novel application for unsupervised methods.

This work aims to investigate the possibility to objectively classify the level of PD in diagnosed patients based on their motor performances, thus promoting a personalized and tailored rehabilitation protocol according to the residual capabilities encouraging the Chronic Care Model. The motion was measured by using wearable IMUs, and processed data were compared using different unsupervised machine learning approaches. In particular, in this study we used a "blind" approach, which means that the data was analyzed without any a priori knowledge on the groups. This work provides at least four novel achievements:

- $\quad$ to analyze a comprehensive experimental protocol for a complete motor evaluation of both lower and upper limbs in PD patients using six exercises according to the tasks described in MDS-UPDRS III.

- To evaluate a wide set of extracted features related to the kinematic characteristics of the users that allow examination of spatial, temporal, and frequency parameters of the limbs motion.

- To investigate the performance of unsupervised learning approaches for PD level assessment based on motor performance by comparing three different methods.

- To evaluate the correlation and adherence between data measured by the sensors and clinical scores assigned by the neurologist.

This blind approach allows to investigate: i) how the proposed technology and algorithms are able to discriminate the different level of the pathology in absence of a priori knowledge about the status of the PD patients; ii) similarities and differences about the performance of PD patients.

\section{MATERIALS AND METHODS}

\section{A. Instrumentation}

A wearable inertial system composed by the SensHand and the SensFoot devices was used in this work for acquiring motion data from both upper and lower limbs (Fig. 1). These devices are based on customized boards with dedicated microcontroller (ARM 32-bit Cortex ${ }^{\mathrm{TM}}-\mathrm{M} 3$ CPU, STMicroelectronics) equipped by LSM9DS (9-axis accelerometer, gyroscope, magnetometer module, STMicroelectronics), and $\mathrm{I}^{2} \mathrm{C}$ digital output. They are supplied by rechargeable LiPo batteries and allowed acquiring data at $100 \mathrm{~Hz}$ sampling frequency. While SensFoot is a unique IMU, which is placed on the foot instep, SensHand is composed by
Figure 1. SensHand (A) and SensFoot (B) wearable insertial

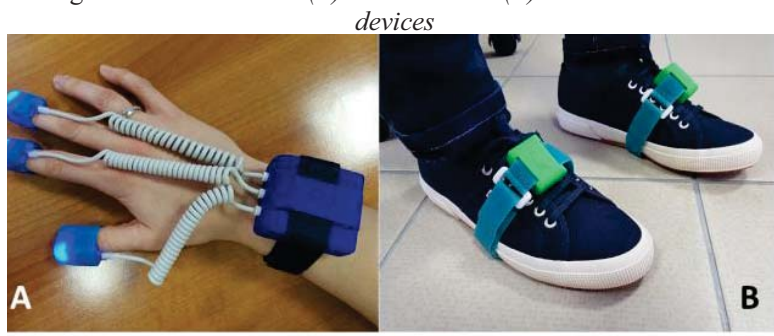

four units, coordinated and synchronized by the CAN bus standard. The module placed on the wrist is the coordinator of SensHand, while the other units are placed on the distal phalanges of thumb, index, and middle fingers. A Bluetooth module enables the wireless communication of the devices with a remote control station [21].

\section{B. Participants}

Thirty people diagnosed with PD were recruited by the clinical staff of the Department of Neurology, Carrara Hospital, Italy. The subjects were 22 males, 8 females, with mean age $\pm \mathrm{SD}=66.8 \pm 9.7$ years old. Exclusion criteria consisted of impairments or disease other than PD that could affect the motor performance of the subjects during daily activities. Moreover, all measurements on PD patients were acquired during a clinically defined ON-state, to avoid complications caused by possible ON/OFF motor fluctuations especially in the most affected patients.

\section{Experimental Protocol}

To assess the subjects' motor performance on the four limbs, the experimental protocol was defined with neurologists, according to the tasks described in the MDSUPDRS III. Particularly, six exercises were selected: thumbforefinger tapping (THFF), hands opening/closing (OPCL), hands pronation/supination (PSUP), hands resting tremor (HRST), toe-tapping (TTHP), and leg agility (HEHE) [22]. They correspond to the MDS-UPDRS tasks 3.4, 3.5, 3.6, 3.17, 3.7 , and 3.8 respectively. Each subject performed all the protocol once, both with right and left sides. The neurologist who observed patients' movements and assigned a score for each exercise according to the MDS-UPDRS III clinically assessed all patients. Then, the sum of the MDS-UPDRS scores assigned by the neurologist for each of the six exercises was calculated. Specifically, according to this score, the subjects were labelled as:

- Mild: if they received a score $\leq 5$;

- Moderate: if they received a score ranging from 6 to 13 ;

- Severe: if they received a score $\geq 14$.

The study was conducted in accordance with the most recent Declaration of Helsinki, and the protocol was approved by the Ethical Committee of Massa and Carrara Local Health Institution with the following reference: No. 311, 18/11/2010. Informed written consent was obtained from all the participants. 
TABLE I. FEATURES LIST. EXTRACTED PARAMETERS FOR EACH EXERCISE ARE CROSSED AND HighLIGHTED IN GREY. ACC = ACCELEROMETER; GYR = GYROSCOPE. $\mathrm{CV}=$ COEFFICIENT OF VARIATION. PSD = POWER SPECTRAL DENSITY

\begin{tabular}{l|c|c|c|c}
\hline & \multicolumn{3}{|c}{ Exercise } & \\
\hline Extracted Feature & $\begin{array}{c}\text { THFF, } \\
\text { OPCL, } \\
\text { PSUP }\end{array}$ & HRST & TTHP & HEHE \\
\hline Number of movements & X & & X & \\
\hline Frequency of movements & $\mathrm{X}$ & & $\mathrm{X}$ & \\
\hline Movement angular excursion & $\mathrm{X}$ & & $\mathrm{X}$ & \\
\hline Opening velocity & $\mathrm{X}$ & & & \\
\hline Closing velocity & $\mathrm{X}$ & & & \\
\hline CV of frequency & $\mathrm{X}$ & & $\mathrm{X}$ & \\
\hline CV of excursion & $\mathrm{X}$ & & $\mathrm{X}$ & \\
\hline Energy expenditure & $\mathrm{X}$ & $\mathrm{X}$ & $\mathrm{X}$ & $\mathrm{X}$ \\
\hline ACC: Average power in PSD & & $\mathrm{X}$ & & $\mathrm{X}$ \\
\hline $\begin{array}{l}\text { ACC: Fundamental } \\
\text { frequency }\end{array}$ & & $\mathrm{X}$ & & $\mathrm{X}$ \\
\hline $\begin{array}{l}\text { ACC: Percentage power in } \\
\text { band [3.5-7.5] Hz }\end{array}$ & & $\mathrm{X}$ & & \\
\hline GYR: Average power in PSD & & $\mathrm{X}$ & & \\
\hline $\begin{array}{l}\text { GYR: Fundamental } \\
\text { frequency }\end{array}$ & & $\mathrm{X}$ & & \\
\hline $\begin{array}{l}\text { GYR: Percentage power in } \\
\text { band [3.5-7.5] Hz }\end{array}$ & & $\mathrm{X}$ & & \\
\hline $\begin{array}{l}\text { ACC: Maximum peak in } \\
\text { PSD }\end{array}$ & & & & $\mathrm{X}$ \\
\hline
\end{tabular}

\section{Extracted Features}

Signals acquired by SensHand and SensFoot were off-line processed applying a fourth-order low-pass digital Butterworth filter. In all exercises, except HRST, cut-off frequency was set equal to $5 \mathrm{~Hz}$ aiming to eliminate highfrequency noise and tremor frequency bands [23], typically identified between $3.5-7.5 \mathrm{~Hz}$ [24], while preserving significant information from the acquired signals. Differently, in HRST, where tremor was analyzed, a fourth-order bandpass digital Butterworth filter was applied with cut-off frequencies equal to $0.5-15 \mathrm{~Hz}$ to avoid the continuous component of the signal and the high-frequency noise. Spatiotemporal and frequency analyses were appropriately conducted implementing custom-made algorithms for the motor data analysis.

\section{E. Data Analysis}

Since motor performance can be highly asymmetrical, especially during the mild stage due to the unilateral onset of the pathology, each side of the 30 PD patients was considered as a separate acquisition, eventually obtaining a dataset composed by 60 instances ( 30 related to the right side, and 30 related to the left side). Then, the instances were divided into three groups according to the level of pathology. Once the dataset has been prepared, a Z-norm was computed to avoid distortion and have a zero mean and a unit standard deviation. Then, three different datasets were defined: one included clinical scores and parameters derived from lower limbs only (FOOT dataset); the second one considered clinical scores and features computed from upper limbs only (HAND dataset); the last one included the complete set of data (FULL dataset combining FOOT + HAND). As proposed in [15], [25] two different analyses were conducted on these datasets.

In the first analysis, the number of clusters $k$ was considered as known. Particularly, in this analysis we consider $\mathrm{k}=2$ and $\mathrm{k}=3$, since two and three hypothetical levels of
Figure 2. Results for three levels of pathology using the full dataset with SOM classifier

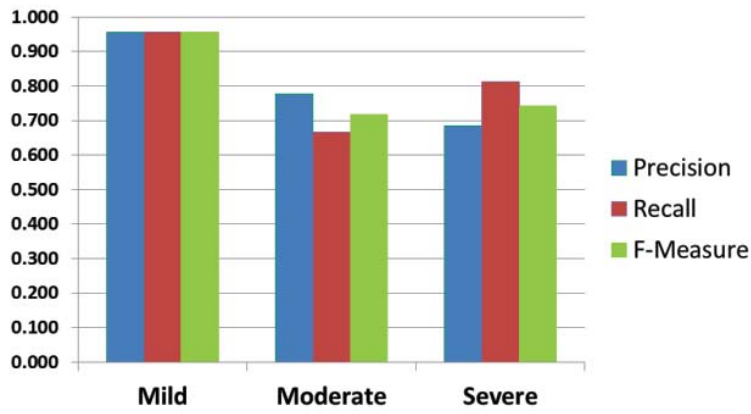

pathology were investigated. The obtained datasets were then analyzed with three unsupervised machine learning algorithms that were used to group instances according to their similarity, thus revealing the implicit relationships in the dataset [26]. Particularly, in this work, the following methods were applied and compared:

- K-Means (KM), which divides the dataset into $\mathrm{k}$ clusters according to the distance between the observation and empirical mean of a cluster [16]. In this work, KM algorithm based on Euclidean distance with three replicates to avoid local minima was implemented.

- Hierarchical Cluster Analysis (HCA), which is a clustering method that tries to build a hierarchy of clusters based on ward linkage [27].

- Self-Organizing Map (SOM), which is a type of artificial network based on competitive learning [28]. This means that neurons compete amongst themselves to be activated, with the result that only one (so-called winning neuron) is activated at any one time. Lateral inhibition connections between the neurons can induce the competition, so the neurons are forced to organize themselves. In this work, SOM was applied with a hexagonal layer topology function.

The Machine Learning and Pattern Recognition Matlab Toolboxes (Matlab®R2016b, The MathWorks, Inc., Natick, MA, USA) were used for the unsupervised analysis.

Secondly, the number of clusters $k$ was considered as unknown looking for the optimal number of clusters that best can divide the dataset. Silhouette index was used as a criterion to assess the cluster validity and to quantitatively analyze and compare the clustering process. A high value of the index indicates that the clusters are compact and well-separated [29]. In particular, this analysis emphasizes whether the proposed blind method could be useful to investigate if the level of the pathology of the patient can be distinguished on the basis of the motor performance.

From a clinical perspective, it would like to evaluate if the proposed wearable system, and related algorithms for data processing, are able to identify different levels of pathology, clustering the patients in groups. 
TABLE II. COMPARATIVE RESUlTS FROM KM, HCA, AND SOM UNSUPERVISED METHODS IN TERMS OF PRECISION, RECALL, SPECIFICITY, ACCURACY, AND F-MEASURE FOR 2 AND 3 CLUSTERS CALCULATED ON FEET, HANDS, AND FULL DATASETS.

\begin{tabular}{|c|c|c|c|c|c|c|c|c|c|c|c|c|c|c|c|}
\hline & \multicolumn{3}{|c|}{ Precision } & \multicolumn{3}{|c|}{ Recall } & \multicolumn{3}{|c|}{ Specificity } & \multicolumn{3}{|c|}{ Accuracy } & \multicolumn{3}{|c|}{ F-measure } \\
\hline & $K M$ & $H C A$ & SOM & $K M$ & $H C A$ & SOM & $K M$ & $H C A$ & SOM & $K M$ & $H C A$ & SOM & $K M$ & $H C A$ & $S O M$ \\
\hline \multicolumn{16}{|l|}{2 Clusters } \\
\hline FOOT & 1.000 & 1.000 & 0.696 & 0.696 & 0.652 & 0.696 & 1.000 & 1.000 & 1.000 & 0.883 & 0.867 & 0.883 & 0.821 & 0.789 & 0.696 \\
\hline HAND & 0.885 & 0.793 & 1.000 & 1.000 & 1.000 & 1.000 & 0.919 & 0.838 & 0.919 & 0.950 & 0.900 & 0.950 & 0.939 & 0.885 & 1.000 \\
\hline FULL & 0.885 & 0.821 & 1.000 & 1.000 & 1.000 & 1.000 & 0.919 & 0.865 & 0.919 & 0.950 & 0.917 & 0.950 & 0.939 & 0.902 & 1.000 \\
\hline \multicolumn{16}{|l|}{3 Clusters } \\
\hline FOOT & 0.693 & 0.660 & 0.687 & 0.653 & 0.553 & 0.638 & 0.815 & 0.770 & 0.804 & 0.650 & 0.567 & 0.633 & 0.672 & 0.601 & 0.662 \\
\hline HAND & 0.756 & 0.806 & 0.756 & 0.754 & 0.812 & 0.754 & 0.867 & 0.902 & 0.867 & 0.750 & 0.817 & 0.750 & 0.755 & 0.809 & 0.755 \\
\hline FULL & 0.790 & 0.806 & 0.806 & 0.796 & 0.812 & 0.812 & 0.899 & 0.902 & 0.906 & 0.800 & 0.817 & 0.817 & 0.793 & 0.809 & 0.809 \\
\hline
\end{tabular}

\section{F. Evaluation}

The performances of the algorithms were evaluated with an external criterion [30] by comparing the output with the a priori knowledge on the level of the pathology. In case of two groups, the classes were labelled as mild vs. advanced (which included both moderate and severe patients), while for threegroup classification, the classes were named mild, moderate, and severe. The true and the predicted labels were organized into confusion matrices and the accuracy, specificity, recall, precision, and F-measure were used to compare and discuss the performances. A comparative analysis of the obtained results considering the different feature arrays and the different number of clusters was performed in order to study the most accurate system for supporting the neurologist in the objective clinical evaluation of the patient.

Firstly, Principal Components Analysis (PCA) was applied in order to reduce the number of features in FULL dataset, thus improving the visualization of the feature space. According to the Kaiser Rule, only components with eigenvalues greater than 1 were considered significant. Sammon's Map was then applied for a further reduction to obtain the two-dimensional representation of data. Particularly, as presented in [19], [31], both approaches are applied to reduce the number of features and improve the visualization of the feature space.

Correlation between data acquired by the IMU devices and clinical scores directly assigned to the patients by the neurologist during the experimental tests was investigated applying a linear regression analysis to examine the adherence of technological solution measurements with clinician's evaluation. MDS-UPDRS III scores were used as the dependent variable and extracted features were the independent variables. Correlation was analyzed for FOOT, HAND, and FULL datasets. Linear regression coefficient $\left(\mathrm{R}^{2}\right)$ and $\mathrm{p}$-value were used as evaluation metrics of this analysis.

\section{RESULTS}

This section reports the results obtained from the "blind" motor performance assessment using three different unsupervised learning approaches (KM, HCA, SOM) on the three different datasets (FOOT, HAND, FULL), providing results for both 2- and 3-group clustering.

Thirty-one features were extracted from upper limbs, and 10 were obtained from lower limbs (see Table I). The final dataset is composed of 60 instances described by 41 features extracted from the six exercises. As concern the level of pathology, 23 instances were considered as mild, 21 instances as moderate, and 16 as severe.

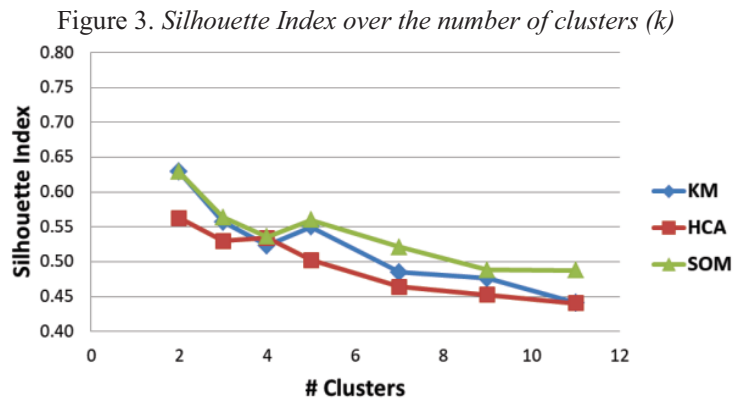

The a posteriori evaluation results are reported in Table II considering FOOT and HAND datasets separately and all together (FULL system), for both $\mathrm{k}=2$ and $\mathrm{k}=3$ clusters analyses. Results from FOOT are the worst, while the FULL dataset allowed to achieve the best performance according to the evaluation metrics. Focusing on full dataset, the comparative evaluation among the three applied unsupervised learning revealed that SOM achieved the best results for both $\mathrm{k}=2$ clusters and $\mathrm{k}=3$ clusters analyses. In particular, for 2 classes (i.e., mild vs. advanced PD) the ability to distinguish the two cohorts of PD patients was excellent (recall equal to $1.000,0.919$ specificity, 0.950 accuracy). Good results were achieved also for 3 clusters investigation ( 0.812 recall, 0.906 specificity, 0.817 accuracy). The complete results are reported in Table II.

Fig. 2 depicts the precision, recall and F-measure of the SOM classifier over the three levels of pathology. The mild PD was the best identified group (precision, recall and Fmeasure $>0.900$ ), whereas moderate and severe PD could be mutually confused in some cases, thus the performance are lower. As concern the analysis with $k$ unknown only the FULL dataset was used into the analysis since it was the one which reached the best accuracy. The Silhouette index underlined that $\mathrm{k}=2$ optimizes the clusters separation for all the unsupervised approaches with an average value equal to 0.61 (Fig. 3). Instead, $\mathrm{k}=3$ has an average index value equal to 0.55 .

A bi-dimensional representation of data is shown in Fig. 4, according to dimensionality reduction achieved by applying a sequence of PCA and Sammon Map. PCA allowed identifying 9 principal components, according to the Kaiser rule, which included $82.3 \%$ of the total variance of the original dataset. Then, Sammon Map enabled the combination of these components in order to obtain a further dimensionality reduction, reaching 2 components able to describe the dataset. Results of 3-group clustering were also reported in the figure with circles, to easily understand data distribution based on the 


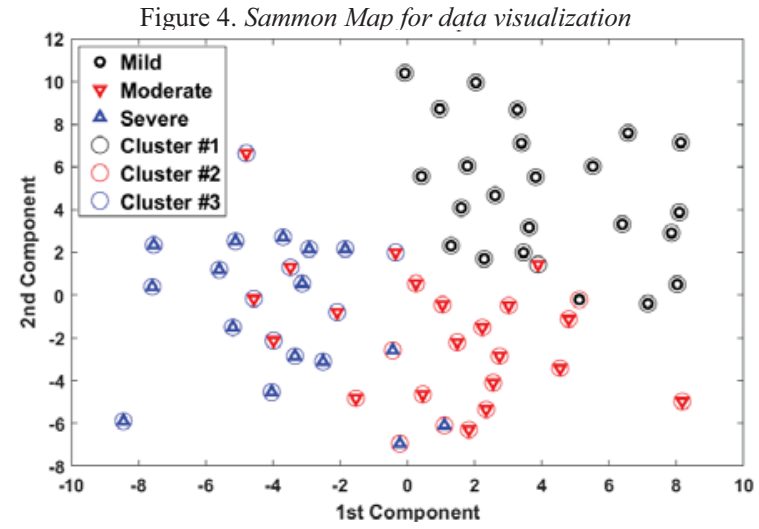

true classes, and the performance of the SOM learning to identify three different clusters associable to the different severity of PD. From a visual inspection perspective, these results confirm that the mild group is well discriminated, while some uncertainties concerning the division between moderate and severe groups. These results confirm the ones reported in Figure 2.

Finally, results from correlation studies are reported in Table III. $\mathrm{R}^{2}$ and related $\mathrm{p}$-value were calculated for each dataset by using linear regression analysis. High correlation values were found between features extracted by IMU devices and clinical scores assigned by the neurologist, which are currently the "gold standard" for PD diagnosis and assessment. Particularly, the FULL dataset achieved the highest correlation coefficient.

\section{DisCUSSION}

This work aimed to investigate the motor performance of PD patients using a blind approach based on unsupervised learning. The aim is the objective assessment of PD patients for identifying different levels of pathology, evaluating also the adherence with the neurologist's evaluation.

The protocol applied was based on six MDS-UPDRS III tasks. Inertial data collected were processed through ad hoc algorithms for features extraction. Then, unsupervised machine learning analysis was applied to investigate whether it could be possible to discriminate different levels of PD on the basis of motion analysis, without a priori knowledge about the clinical status of the patients. Multiple comparisons were implemented and analyzed in this work, using three different unsupervised approaches (i.e., KM, HCA, SOM) tested on three datasets (i.e., FOOT, HAND, FULL), for two-group or three-group classification.

Results from FOOT dataset were the worst, while the FULL dataset allowed to obtain the best performance in terms of evaluation metrics. The modest results from FOOT can be due to the specific nature of the patients involved in the study. Furthermore, from a technical point of view, the FOOT dataset was represented by two exercises only, computing 10 parameters, which can contain limited information about the motion performance of PD patients.

Since the full dataset achieved the best results for both twoand three-group classification, this work promotes the use of a comprehensive protocol, which included tasks from both upper and lower limbs for a complete assessment of PD motor
TABLE III. CORRELATION BETWEEN EXTRACTED FEATURES AND CLINICAL SCORES BY USING LINEAR REGRESSION ANALYSIS

\begin{tabular}{|c|c|c|}
\hline Dataset & $\mathbf{R}^{\mathbf{2}}$ & p-value \\
\hline FOOT & 0.721 & $1.71 \mathrm{E}-10$ \\
\hline HAND & 0.955 & $4.81 \mathrm{E}-12$ \\
\hline FULL & 0.971 & $6.80 \mathrm{E}-08$ \\
\hline
\end{tabular}

performance. Considering PD has usually asymmetric onset, becoming bilateral during advanced stages, the FULL approach has the advantage to simultaneously monitor the performance of all limbs. Concerning the full dataset, the best results were obtained for two-class classification with SOM (1.000 recall, precision and F-measure, 0.950 accuracy, 0.919 specificity). Classifying PD patients in two groups (i.e., mild vs. advanced patients) according to motion analysis results was already studied by Butt et al. [22]. However, in such work, supervised approaches were used, achieving the best performance with a Neural Network classifier obtaining accuracy equal to 0.83 that is worse than the result of this study.

Good results were achieved also for three-group clustering with SOM (0.812 recall, 0.817 accuracy, 0.906 specificity), where the main difficulties for classification concerned the separation between moderate and severe patients, as shown in Fig. 4 where Mild group appear easily separable, while Moderate and Severe instances are partially overlapped. However, currently, this seems the first work in literature that tries to implement a multi-class clustering based on PD motion analysis using unsupervised methods with both upper and/or lower limbs. Overall, the obtained results are promising to sustain the application of the proposed system in clinical practice for supporting clinicians in the quantitative assessment of PD diagnosis.

Correlation analyses between extracted features from inertial data and MDS-UPDRS III scores provided excellent results, in particular for HAND and FULL datasets $\left(\mathrm{R}^{2}\right.$ equal to 0.955 , and 0.971 respectively). This achievement confirmed that the system could really support the clinical diagnosis of PD, objectifying the MDS-UPDRS III, which is currently the "gold standard" for PD assessment. The high correlation values obtained, indeed, are significant of good adherence between parameters measured by SensHand and SensFoot devices and observations derived by neurologist's visual inspection.

According to these results, the use of unsupervised approaches resulted appropriate for identification of different classes of PD patients related to the different level of pathology. The system is able to correctly group the instances related to motor performance. These results suggest the potential use of the proposed system (protocol, extracted features and devices) as a support tool in clinical application for the correct management of the PD.

\section{CONCLUSION}

This study proposed a novel approach based on unsupervised learning to support neurologists in the objective assessment of PD on the basis of quantitative analysis of patients motor performance. In particular, the unsupervised 
methods, and the blind approach used, allowed to automatically identify separate clusters in which to group PD patients without a priori knowledge about their clinical evaluation. Two-group and three-group clustering were obtained with good accuracy if comparing results from unsupervised learning (that used only data from motor acquisitions) with the true labels gave out to the patients on the basis of clinical scores assigned by the neurologist. Further, excellent adherence to clinical assessment obtained with the retrospective analysis made the system suitable for objectifying evaluations based on the clinical scale. Thus, such system can enable the motor capabilities assessment of PD patients, allowing to identify the best rehabilitative and assistive treatment for each of them. Future works will consolidate these results with the enlargement of the number of involved patients (as well as the number of exercises in the protocol), aiming at increasing the accuracy of the patients assessment to improve the quality of care for PD, identifying personalized care-rehabilitation plan.

\section{REFERENCES}

[1] E. R. Dorsey and B. R. Bloem, "The Parkinson Pandemic-A Call to Action," JAMA Neurol., vol. 75, no. 1, p. 9, Jan. 2018.

[2] S. von Campenhausen, Y. Winter, A. Rodrigues e Silva, C. Sampaio, E. Ruzicka, P. Barone, W. Poewe, A. Guekht, C. Mateus, K. P. Pfeiffer, K. Berger, J. Skoupa, K. Bötzel, S. Geiger-Gritsch, U. Siebert, M. Balzer-Geldsetzer, W. H. Oertel, R. Dodel, and J. P. Reese, "Costs of illness and care in Parkinson's Disease: An evaluation in six countries," Eur. Neuropsychopharmacol., vol. 21, no. 2, pp. 180-191, 2011

[3] R. B. Postuma, D. Berg, M. Stern, W. Poewe, C. W. Olanow, W Oertel, J. Obeso, K. Marek, I. Litvan, A. E. Lang, G. Halliday, C. G. Goetz, T. Gasser, B. Dubois, P. Chan, B. R. Bloem, C. H. Adler, and G. Deuschl, "MDS clinical diagnostic criteria for Parkinson's disease," Mov Disord, 2015.

[4] J. Kassubek, "Diagnostic procedures during the course of Parkinson's Disease," Basal Ganglia, vol. 4, no. 1, pp. 15-18, 2014.

[5] V. J. Barr, S. Robinson, B. Marin-Link, L. Underhill, A. Dotts, D Ravensdale, and S. Salivaras, "The expanded Chronic Care Model: an integration of concepts and strategies from population health promotion and the Chronic Care Model.," Hosp. Q., vol. 7, no. 1, pp. 73-82, 2003

[6] G. Turchetti, S. Micera, F. Cavallo, L. Odetti, and P. Dario, "Technology and Innovative Services," IEEE Pulse, vol. 2, no. 2, pp 27-35, Mar. 2011.

[7] T. Bodenheimer, E. Wagner, and K. Grumbach, "Improving primary care for patients with chronic illness," JAMA, vol. 288, no. 14, pp. 1775-1779, 2002.

[8] M. El-Gohary, S. Pearson, J. McNames, M. Mancini, F. Horak, S Mellone, and L. Chiari, "Continuous monitoring of turning in patients with movement disability," Sensors, vol. 14, no. 1, pp. 356369,2014

[9] E. Rovini, C. Maremmani, and F. Cavallo, "How wearable sensors can support parkinson's disease diagnosis and treatment: A systematic review," Front. Neurosci., vol. 11, no. OCT, 2017.

[10] J. M. Fisher, N. Y. Hammerla, T. Ploetz, P. Andras, L. Rochester, and R. W. Walker, "Unsupervised home monitoring of Parkinson's disease motor symptoms using body-worn accelerometers," Park. Relat. Disord., vol. 33, pp. 44-50, 2016.

[11] N. Kostikis, M. Arnaoutoglou, and C. Kotsavasiloglou, "A Smartphone-based tool for assessing Parkinsonian hand tremor," $J$. Biomed. Heal. Informatics, vol. 19, no. 6, pp. 1835-1842, 2015.

[12] S. Patel, K. Lorincz, R. Hughes, N. Huggins, J. Growdon, D. Standaert, M. Akay, J. Dy, M. Welsh, P. Bonato, and S. Member, "Monitoring motor fluctuations in patients with Parkinson's disease using wearable sensors," IEEE Trans. Inf. Technol. Biomed., vol. 13, no. 6 , pp. $864-873,2009$
[13] S. Reinfelder, R. Hauer, J. Barth, J. Klucken, and B. M. Eskofier, "Timed Up-and-Go phase segmentation in Parkinson's disease patients using unobtrusive inertial sensors," in 37th Ann. Int. Conf. of the IEEE Engineering in Medicine and Biology Society (EMBC), 2015, pp. 5171-5174.

[14] S. V. Perumal and R. Sankar, "Gait and tremor assessment for patients with Parkinson's disease using wearable sensors," ICT Express, vol. 2, no. 4, pp. 168-174, 2016.

[15] A. Moschetti, L. Fiorini, D. Esposito, P. Dario, and F. Cavallo, "Toward an Unsupervised Approach for Daily Gesture Recognition in Assisted Living Applications," IEEE Sens. J., vol. 17, no. 24, pp. 8395-8403, Dec. 2017.

[16] D. Biswas, A. Cranny, N. Gupta, K. Maharatna, J. Achner, J. Klemke, M. Jöbges, and S. Ortmann, "Recognizing upper limb movements with wrist worn inertial sensors using k-means clustering classification," Hum. Mov. Sci., vol. 40, pp. 59-76, 2015.

[17] L. Kaufman and P. J. Rousseeuw, Finding groups in data : an introduction to cluster analysis. Wiley-Interscience, 1990

[18] G. Sprint, D. J. Cook, and M. Schmitter-edgecombe, "Unsupervised Detection and Analysis of Changes in Everyday Physical Activity Data," J. Biomed. Inform., no. 63, pp. 54-65, 2016.

[19] L. Fiorini, F. Cavallo, P. Dario, A. Eavis, and P. Caleb-Solly, "Unsupervised Machine Learning for Developing Personalised Behaviour Models Using Activity Data," Sensors, vol. 17, no. 5, p. 1034, May 2017.

[20] W. El Ansari, D. Ssewanyana, and C. Stock, "Behavioral Health Risk Profiles of Undergraduate University Students in England, Wales, and Northern Ireland: A Cluster Analysis.," Front. public Heal., vol. 6, p. 120, 2018.

[21] F. Cavallo, D. Esposito, E. Rovini, M. Aquilano, M. C. Carrozza, P. Dario, C. Maremmani, and P. Bongioanni, "Preliminary evaluation of SensHand V1 in assessing motor skills performance in Parkinson disease," in 2013 IEEE 13th International Conference on Rehabilitation Robotics (ICORR), 2013, pp. 1-6.

[22] A. H. Butt, E. Rovini, D. Esposito, G. Rossi, C. Maremmani, and F. Cavallo, "Biomechanical parameter assessment for classification of Parkinson's disease on clinical scale," Int. J. Distrib. Sens. Networks, vol. 13, no. 5, p. 155014771770741 , May 2017.

[23] J.-W. Kim, Y. Kwon, Y.-M. Kim, H.-Y. Chung, G.-M. Eom, J.-H. Jun, J.-W. Lee, S.-B. Koh, B. K. Park, and D.-K. Kwon, "Analysis of lower limb bradykinesia in Parkinson's disease patients," Geriatr. Gerontol. Int., vol. 12, no. 2, pp. 257-264, Apr. 2012.

[24] G. Rigas, a. T. Tzallas, M. G. Tsipouras, P. Bougia, E. E. Tripoliti, D. Baga, D. I. Fotiadis, S. G. Tsouli, and S. Konitsiotis, "Assessment of tremor activity in the Parkinson's Disease using a set of wearable sensors," IEEE Trans. Inf. Technol. Biomed., vol. 16, no. 3, pp. 478487, 2012.

[25] Y. Kwon, K. Kang, and C. Bae, "Unsupervised learning for human activity recognition using smartphone sensors," Expert Syst. Appl., vol. 41, no. 14, pp. 6067-6074, Oct. 2014.

[26] F. Li and S. Dustdar, "Incorporating Unsupervised Learning in Activity Recognition," in Activity Context Representation, 2011.

[27] L. Rokach, "A survey of clustering algorithms," in Data mining and knowledge discovery handbook, Springer, 2009, pp. 269-298.

[28] J. A. Bullinaria, "Self organizing maps: fundamentals," Introd. to Neural, 2004

[29] P. J. Rousseeuw, "Silhouettes: a graphical aid to the interpretation and validation of cluster analysis," J. Comput. Appl. Math., vol. 20, pp. 53-65, 1987

[30] A. K. Jain and R. C. Dubes, "Algorithms for clustering data," 1988.

[31] S. Patel, D. Sherrill, R. Hughes, T. Hester, N. Huggins, T. LieNemeth, D. Standaert, and P. Bonato, "Analysis of the severity of dyskinesia in patients with Parkinson's disease via wearable sensors," in International Conference on Wearable and Implantable Body Sensor Networks (BSN) Workshops, 2006, pp. 123-126. 